\title{
EXTENDED EIGENVALUES AND THE VOLTERRA OPERATOR
}

\author{
ANIMIKH BISWAS, ALAN LAMBERT \\ 9201 University City Blvd, Department of Mathematics, UNC Charlotte, Charlotte, NC 28223, USA \\ e-mail:abiswas@uncc.edu and allamber@email.uncc.edu \\ and SRDJAN PETROVIC \\ Department of Mathematics, Western Michigan University, Kalamazoo, MI 49008, USA \\ e-mail: srdjan.petrovic@wmich.edu
}

(Received 5 April, 2001; accepted 10 October, 2001)

\begin{abstract}
In this paper we consider the integral Volterra operator on the space $L^{2}(0,1)$. We say that a complex number $\lambda$ is an extended eigenvalue of $V$ if there exists a nonzero operator $X$ satisfying the equation $X V=\lambda V X$. We show that the set of extended eigenvalues of $V$ is precisely the interval $(0, \infty)$ and the corresponding eigenvectors may be chosen to be integral operators as well.
\end{abstract}

2000 Mathematics Subject Classification. Primary 47A65; Secondary 47B49, 47B38.

1. Introduction and preliminaries. Let $\mathcal{H}$ be a complex Hilbert space. Denote by $\mathcal{L}(\mathcal{H})$ the algebra of all bounded linear operators on $\mathcal{H}$. Consider an operator $A$ in $\mathcal{L}(\mathcal{H})$. If $X \in \mathcal{L}(\mathcal{H})$ it can happen that there is a nonzero operator $Y$ such that

$$
X A=A Y .
$$

If we denote by $\mathcal{E}_{A}$ the set of all $X$ for which there exists an operator $Y$ satisfying (1.1), then it is easy to see that $\mathcal{E}_{A}$ is an algebra. Furthermore, if $A$ has dense range, one can define the map $\Phi_{A}: \mathcal{E}_{A} \rightarrow \mathcal{L}(\mathcal{H})$ by $\Phi_{A}(X)=Y$. One can easily see that $\Phi_{A}$ is an algebra homomorphism, and we shall verify shortly that it is in fact a closed (generally unbounded) linear transformation.

When $Y=\lambda X$, for some complex number $\lambda$, equation (1.1) becomes

$$
X A=\lambda A X .
$$

Clearly, a pair $(X, \lambda)$ in $\mathcal{L}(\mathcal{H}) \backslash(0) \times \mathbb{C}$ satisfies (1.2) if and only if $\lambda$ is an eigenvalue for $\Phi_{A}$ and $X$ is an eigenvector for $\Phi_{A}$. An eigenvalue of $\Phi_{A}$ will be referred to as an extended eigenvalue of $A$.

One knows that, when $\lambda=1$, equation (1.2) can be used to obtain information about the operator $X$ based on the properties of the operator $A$. In particular, a famous result of Lomonosov [5] asserts that if $A$ is compact then $X$ must have a

The third author was supported in part by the FRACASF grant from the Western Michigan University. 
nontrivial hyperinvariant subspace. In fact, the whole commutant $\{A\}^{\prime}$ of $A$ has a common nontrivial invariant subspace. Later, it was shown independently by $\mathrm{S}$. Brown [3] and Kim, Pearcy, and Shields [7] that if $A$ is compact and $X$ satisfies (1.2), for any complex $\lambda$, then $X$ has a nontrivial hyperinvariant subspace. This extension naturally leads to the question as to whether there is an algebra $\mathcal{A}$ that properly contains $\{A\}^{\prime}$ and which, under specific conditions, has an invariant subspace. Such an algebra has been introduced in [6] and it was shown there that it contains not only those operators that commute with $A$ but also operators that satisfy (1.2) for some $|\lambda| \leq 1$. Furthermore, if $A$ is compact, then this algebra has a nontrivial invariant subspace. Of course, if $\mathcal{A}=\{A\}^{\prime}$ this is just Lomonosov's theorem. Therefore it is of interest to find out whether the inclusion

$$
\{A\}^{\prime} \subset \mathcal{A}
$$

is proper. It was established in [6] that this happens when the spectral radius of $A$ is positive. Thus, it remains to consider the case in which $A$ is compact and quasinilpotent.

It is the purpose of this paper to make a first step in this direction by showing that the inclusion (1.3) is proper when $A$ is a specific compact, quasinilpotent operator. More precisely, let $\mathcal{H}=L^{2}(0,1)$, and $A=V$, the Volterra integral operator on $L^{2}(0,1)$, defined by

$$
V f(x)=\int_{0}^{x} f(t) d t .
$$

We shall show that the set of extended eigenvalues of the Volterra operator $V$ is precisely the set $(0, \infty)$. Moreover, we shall show that for each such an extended eigenvalue $\lambda$, the appropriate eigenvector can be found in the class of integral operators. In other words, for each $\lambda>0$, the equation

$$
X V=\lambda V X
$$

has a nonzero integral operator as a solution.

The organization of the paper is as follows. In Section 2, we show that if $\lambda$ does not belong to $(0, \infty)$ then it cannot be an eigenvalue of $\Phi_{V}$. As an application of our method we show as well that, for $\lambda \neq 1$, the operators $V$ and $\lambda V$ are not quasisimilar.

As possible candidates for a solution of (1.4) we consider three classes of operators: operators of multiplication, integral operators, and composition operators. Sections 3, 4, and 5, respectively, are dedicated to these classes. We shall show that if $\lambda \in(0, \infty)$ then it is an eigenvalue of $\Phi_{V}$; that is, in this case (1.4) has nontrivial solutions. Finally, in Section 6 we present some open problems.

Once we establish the fact that the spectrum of $\Phi_{V}$ is unbounded it will follow that $\Phi_{V}$ is an unbounded map. Here we show that, whenever $A$ is an operator in $\mathcal{L}(\mathcal{H})$ with dense range, $\Phi_{A}$ is a closed linear map.

Theorem 1. For $A \in \mathcal{L}(\mathcal{H})$ with dense range, the map $\Phi_{A}$ is a closed map on $\mathcal{E}_{A}$.

Proof. We need to show that if $\left\{T_{n}\right\}$ is a norm convergent sequence in $\mathcal{E}_{A}$ converging to an operator $T$, and if $\Phi_{A}\left(T_{n}\right)$ converges in norm to some $S \in \mathcal{L}(\mathcal{H})$, then $S \in \mathcal{E}_{A}$ and $S=\Phi_{A}(T)$. Denote $S_{n}=\Phi_{A}\left(T_{n}\right)$. Then 


$$
T_{n} A=A S_{n} .
$$

Clearly $T_{n} A \rightarrow T A$ and $A S_{n} \rightarrow A S$, as $n \rightarrow \infty$, so that (1.5) yields $T A=A S$, which is equivalent to $\Phi_{A}(T)=S$.

2. Point spectrum of $\Phi_{V}$. In this section, we take the first step to determine the point spectrum of $\Phi_{V}$. In particular, we show that equation (1.4) has no nonzero operator $X$ as a solution if $\lambda \in \mathbb{C} \backslash(0, \infty)$.

We start by noticing that 0 cannot be an eigenvalue of $\Phi_{V}$. This follows easily from the observation that $V$ has dense range in $L^{2}(0,1)$. (In fact, the range of $V$ consists of all absolutely continuous functions which are zero at the origin.) Since we can assume that $\lambda \neq 0$, equation (1.4) is equivalent to the equation

$$
V X=\beta X V
$$

where $\beta=1 / \lambda$. We show in this section that the operator equation (2.1) has no nontrivial solution in the case in which $\beta \in \mathbb{C} \backslash(0, \infty)$. This will enable us to conclude that the point spectrum of $\Phi_{V}$ is a subset of $(0, \infty)$. In subsequent sections, it will be shown that, when $\lambda \in(0, \infty)$, equation (1.4) does indeed have nontrivial solutions. It will then follow that the point spectrum of $\Phi_{V}$ is $(0, \infty)$.

As an application of the techniques that we introduce in this section we also show that, for $\beta \neq 1$, the operators $V$ and $\beta V$ are not quasisimilar.

As usual, when $E$ is a set, the symbol $\chi_{E}$ denotes the characteristic function of $E$; i.e., $\chi_{E}(x)=1$ if $x \in E$ and $\chi_{E}(x)=0$ if $x \notin E$. In what follows, whenever necessary, we regard $L^{2}(0,1)$ as a closed subspace of $L^{2}(-\infty, \infty)$ consisting of all those (equivalence classes of) functions vanishing outside of $(0,1)$. Let $\mathcal{D}=\{f: f$ is absolutely continuous and $f(0)=0\}$. Also, let $D$ denote the unbounded operator with domain $\mathcal{D}$ defined by $D f=\frac{d}{d x} f$ for $f \in \mathcal{D}$. In all equations involving $D$ we assume that they are restricted to $\mathcal{D}$. Thus, we write $V D=D V=I$. In order to understand better the spectral behavior of these operators it is useful to consider a certain semigroup of operators. Namely, for each $t \geq 0$, let $S_{t}$ be the operator on $L^{2}(0,1)$ defined by

$$
S_{t} f(x)=\chi_{[t, \infty) \cap[0,1]}(x) f(x-t) .
$$

It is easy to verify that $\left\{S_{t}: 0 \leq t<\infty\right\}$ is a strongly continuous semigroup on $L^{2}(0,1)$. Notice that, for $t \geq 1, S_{t}=0$. The following result is going to be used in the proof of Theorem 3. For its proof as well as the perusal of this circle of ideas we recommend [1].

Theorem 2. The infinitesimal generator of $S_{t}$ is $-D$. Moreover, for all $z \in \mathbb{C},(z+D)^{-1}$ exists as a bounded operator and

$$
(z+D)^{-1}=\int_{0}^{1} e^{z t} S_{t} d t .
$$

We now state and prove the main theorem in this section. 
Theorem 3. Suppose that $\beta \in \mathbb{C} \backslash(0, \infty)$. Then there is no nonzero operator $T$ on $L^{2}(0,1)$ satisfying the equation $V T=\beta T V$.

Proof. First note that since $V$ is injective, there is no nontrivial solution of the equation $V T=\beta T V$ for $\beta=0$ and henceforth for the rest of this section, we assume $\beta \neq 0$.

Let $T$ be an operator on $L^{2}(0,1)$ satisfying the equation

$$
V T=\beta T V
$$

for some $\beta \in \mathbb{C}$. Since $\mathcal{D}$ is precisely the range of $V$, equation (2.4) implies that $T(\mathcal{D}) \subset \mathcal{D}$. Consequently, equation (2.4) is equivalent to

$$
T D=\beta D T \text {. }
$$

Let $z \in \mathbb{C}$. Then

$$
T(z+D)=(z+\beta D) T=\beta\left(\frac{z}{\beta}+D\right) T
$$

Since $z$ was arbitrary, the previous equation is equivalent to $T(\beta z+D)=\beta(z+D) T$. Therefore, $(z+D)^{-1} T=\beta T(\beta z+D)^{-1}$. Using Theorem 2, we obtain

$$
\int_{0}^{1} e^{z t} S_{t} T d t=\beta \int_{0}^{1} e^{\beta z t} T S_{t} d t
$$

Let $u$ and $v$ belong to $L^{2}(0,1)$. Define $f(t)=\left(S_{t} T u, v\right)$ and $g(t)=\beta\left(T S_{t} u, v\right)$. Here, $(\cdot, \cdot)$ denotes the standard inner product in $L^{2}(0,1)$. Note that since $S_{t}$ is a strongly continuous semigroup, $f$ and $g$ are continuous functions on $[0,1]$. From the definition of the functions $f, g$ and (2.6) it follows that $\int_{0}^{1} e^{z t} f(t) d t=\int_{0}^{1} e^{\beta z t} g(t) d t$. Next, we expand both sides of this equation as power series in $z$ and we notice that both series converge uniformly in $t$, for each $z \in \mathbb{C}$. Thus we can integrate the series term by term. Comparing coefficients in these series we obtain

$$
\int_{0}^{1} t^{n} f(t) d t=\int_{0}^{1} \beta^{n} t^{n} g(t) d t \quad(n \geq 0) .
$$

It follows that, for every polynomial $p$, we have

$$
\int_{0}^{1} p(t) f(t) d t=\int_{0}^{1} p(\beta t) g(t) d t .
$$

Now suppose that $\beta \in \mathbb{C} \backslash[0, \infty)$. Let $S=\{\beta t: 0 \leq t \leq 1\} \cup[0,1]$. Define a function $h$ on $S$ by $h(\beta t)=\overline{g(t)}, t \in[0,1]$ and $h=0$ otherwise. Then $h \in L^{2}(S)$ with respect to the standard Lebegue measure on $S$. By Mergelyan's theorem (cf. [8, p. 390]), there exists a sequence of polynomials $p_{n}$ on $S$ converging to $h$ in $L^{2}(S)$. But then, $\int_{0}^{1} p_{n}(t) f(t) d t \rightarrow 0$ and $\int_{0}^{1} p_{n}(\beta t) g(t) d t \rightarrow\|g\|^{2}$ as $n \rightarrow \infty$. In view of (2.7), this shows that $g=0$ in $L^{2}(0,1)$ and, since $g$ is continuous, this means that $g(t)=0$, for all $t$ in $[0,1]$. In particular, $g(0)=\beta(T u, v)=0$. Since $\beta \neq 0$ and $u, v$ are arbitrary in $L^{2}(0,1)$ it follows that $T=0$. 
We now show that for $\beta \neq 1$ the operators $V$ and $\beta V$ are not quasisimilar. First, we need the following lemma. Once again, $S_{t}$ is the semigroup defined in (2.2).

Lemma 1. Let $\beta>0$ and let $T$ be an operator on $L^{2}(0,1)$. Then $V T=\beta T V$ if and only if $S_{t} T=T S_{t / \beta}$, for all $t \geq 0$.

Proof. Suppose that $V T=\beta T V$. Just as in the proof of Theorem 3 let $u$ and $v$ belong to $L^{2}(0,1)$. Define $f(t)=\left(S_{t} T u, v\right)$ and $g(t)=\beta\left(T S_{t} u, v\right)$.

First we consider the case when $0<\beta \leq 1$. Let $h \in L^{2}(0,1)$. Using the fact that polynomials are dense in $L^{2}(0,1)$ and $(2.7)$, we have

$$
\int_{0}^{1} h(t) f(t) d t=\int_{0}^{1} h(\beta t) g(t) d t=\frac{1}{\beta} \int_{0}^{1} \chi_{[0, \beta]}(t) h(t) g\left(\frac{t}{\beta}\right) d t .
$$

This implies that $f(t)=(1 / \beta) \chi_{[0, \beta]}(t) g(t / \beta)$ a. e. on $[0,1]$. Since $f(t)=0$ for $t \geq 1$ and $g(t / \beta)=0$ for $t \geq \beta$, it follows that $f(t)=(1 / \beta) g(t / \beta)$ for a. e. $t$ and, thus,

$$
\left(S_{t} T u, v\right)=\left(T S_{t / \beta} T u, v\right) .
$$

Since $u$ and $v$ are arbitrary, it follows that $S_{t} T=T S_{t / \beta}$ for all $t \geq 0$ and $0<\beta \leq 1$.

Next we turn our attention to the case $\beta>1$. Once again using (2.7) we have that, for any polynomial $p$,

$$
\int_{0}^{1} p(t) f(t) d t=\int_{0}^{1} p(\beta t) g(t) d t=\frac{1}{\beta}\left(\int_{0}^{1} p(t) g\left(\frac{t}{\beta}\right) d t+\int_{1}^{\beta} p(t) g\left(\frac{t}{\beta}\right) d t\right) .
$$

Let $h$ be an arbitrary function in $L^{2}(0,1)$ and recall that $h$ can be viewed as a function in $L^{2}(0, \beta)$ such that $h=0$ a. e. on $(1, \beta)$. Let $\left\{p_{n}\right\}$ be a sequence of polynomials converging in $L^{2}(0, \beta)$ to $h$. Then,

$$
\int_{0}^{1} p_{n}(t) f(t) d t \rightarrow \int_{0}^{1} h(t) f(t) d t
$$

and

$$
\frac{1}{\beta} \int_{0}^{1} p_{n}(t) g\left(\frac{t}{\beta}\right) d t+\frac{1}{\beta} \int_{1}^{\beta} p_{n}(t) g\left(\frac{t}{\beta}\right) d t \longrightarrow \frac{1}{\beta} \int_{0}^{1} h(t) g\left(\frac{t}{\beta}\right) d t+0 .
$$

Since $h$ is an arbitrary function in $L^{2}(0,1)$, using equation (2.8) with $p_{n}$ in place of $p$, it follows that $f(t)=(1 / \beta) g(t / \beta)$ a. e. on $[0,1]$ and, as before, it means that $S_{t} T=T S_{t / \beta}$, for all $t \geq 0$.

Suppose now that $S_{t} T=T S_{t / \beta}$, for all $t \geq 0$. It was shown in the proof of Theorem 3 that the equality $V T=\beta T V$ is equivalent to (2.6). Again, we consider separately the cases $0<\beta \leq 1$ and $\beta>1$.

Let $0<\beta \leq 1$. Using the assumption and a simple change of variables we get that

$$
\int_{0}^{1} e^{z t} S_{t} T d t=\int_{0}^{1} e^{z t} T S_{t / \beta} d t=\beta \int_{0}^{1 / \beta} e^{\beta z t} T S_{t} d t=\beta \int_{0}^{1} e^{\beta z t} T S_{t} d t
$$

where the last equality follows from the facts that $1 / \beta \geq 1$ and $S_{t}=0$ for $t \geq 1$. 
If $\beta>1$ we start with the right hand side of (2.6). Using an analogous argument we obtain that

$$
\beta \int_{0}^{1} e^{\beta z t} T S_{t} d t=\int_{0}^{\beta} e^{z t} T S_{t / \beta} d t=\int_{0}^{\beta} e^{z t} S_{t} T d t=\int_{0}^{1} e^{z t} S_{t} T d t,
$$

and the lemma is proved.

Recall that two operators $X$ and $Y$ on any two Hilbert spaces $\mathcal{X}$ and $\mathcal{Y}$ respectively are said to be quasisimilar if there exist operators $T_{1}$ from $\mathcal{X}$ to $\mathcal{Y}$ and $T_{2}$ from $\mathcal{Y}$ to $\mathcal{X}$ such that both $T_{1}$ and $T_{2}$ are one to one with dense ranges and such that $T_{1} X=Y T_{1}$ and $X T_{2}=T_{2} Y$. Of course, when $T_{1}$ and $T_{2}$ are equal and invertible, this yields the usual definition of similarity.

Theorem 4. For $\beta \neq 1$, the operators $V$ and $\beta V$ are not quasisimilar.

Proof. If $\beta$ is not a positive real number, the claim follows from Theorem 3 . Now suppose that $\beta>0, \beta \neq 1$ and let $T$ be an operator on $L^{2}(0,1)$ such that $V T=\beta T V$. Then, by Lemma $1, S_{t} T=T S_{t / \beta}$. If $\beta<1$, then

$$
S_{\beta} T=T S_{1}=0 \text {. }
$$

If $\beta>1$, then

$$
0=S_{1} T=T S_{\frac{1}{\beta}}
$$

However, note that $S_{t}=0$ if and only if $t \geq 1$. Therefore, from (2.9) and (2.10) it follows that in case $\beta>0, \beta \neq 1$ the operator $T$ is either not one to one or does not have dense range. As a result, $V$ and $\beta V$ cannot be quasisimilar if $\beta \neq 1$.

3. Operators of multiplication. Let $\varphi \in L^{\infty}(0,1)$. The operator $M_{\varphi}$ on $L^{2}(0,1)$, defined by $\left(M_{\varphi} f\right)(x)=\varphi(x) f(x)$, is called the operator of multiplication by $\varphi$. In this section we are interested in whether $M_{\varphi}$ can satisfy condition (1.4), for some $\varphi$ in $L^{\infty}(0,1)$. The following result shows that the answer to this question is negative.

TheORem 5. Let $\varphi \in L^{\infty}(0,1)$ and let $M_{\varphi}$ be the operator of multiplication by $\varphi$ in $\mathcal{L}\left(L^{2}(0,1)\right)$. If $M_{\varphi} V=\lambda V M_{\varphi}$, then $\varphi=0$ almost everywhere in $(0,1)$

Proof. The equation $M_{\varphi} V=\lambda V M_{\varphi}$ implies that

$$
\varphi(x) \int_{0}^{x} f(t) d t=\lambda \int_{0}^{x} \varphi(t) f(t) d t, \quad \forall f \in L^{2}(0,1), \quad \text { for a. e. } x \in[0,1] .
$$

Equivalently,

$$
\int_{0}^{x} f(t)[\varphi(x)-\lambda \varphi(t)] d t=0
$$


for all $f$ in $L^{2}(0,1)$ and for a. e. $x \in[0,1]$. Hence,

$$
\int_{0}^{1} f(t) \chi_{[0, x]}(t)[\varphi(x)-\lambda \varphi(t)] d t=0
$$

for all $f$ in $L^{2}(0,1)$ and for a. e. $x \in[0,1]$. Notice that the reason that equation (3.2) is valid only almost everywhere is that $\varphi$ is defined only up to sets of measure zero. Due to the separability of $L^{2}(0,1)$ the set of numbers $x$ for which (3.2) holds can be chosen to be the same for any $f \in L^{2}(0,1)$. Thus, the quantifiers on $f$ and on $x$ can and will be taken to be independent. Therefore, for any $x$ satisfying (3.2), we have that $\chi_{[0, x]}(t)[\varphi(x)-\lambda \varphi(t)]=0$ for a. e. $t$, which implies that $\varphi$ is constant a. e. Consequently, $\varphi$ must be a constant function in $L^{\infty}(0,1)$. Furthermore, if $\varphi(x)=C$ then $\chi_{[0, x]}(t)[C-\lambda C]=0$ for a. e. $t$ and, therefore, $C=0$.

Theorem 5 shows that $M_{\varphi}$ cannot be an eigenvector of $\Phi_{V}$. In other words, if $\Phi_{V}^{M}$ is the restriction of $\Phi_{V}$ to the subspace consisting of operators of multiplication, then the point spectrum of $\Phi_{V}^{M}$ consists of $\lambda=1$ alone. Moreover, the proof of Theorem 5 shows that, in this case, $M_{\varphi}$ is an eigenvector for $\Phi_{V}^{M}$ if and only if $\varphi(x)=C$ a. e., for some constant $C$. This leaves open the question about other parts of the spectrum of $\Phi_{V}^{M}$. We conclude this discussion with the following problem. $\Phi_{V}^{M}$

Problem 1. Describe the spectrum of $\Phi_{V}^{M}$. Describe parts of the spectrum of

4. Integral operators. We now investigate whether there exists a nonzero integral operator that satisfies equation (1.4), for some $\lambda>0$. Throughout this section we shall be dealing exclusively with Lebesgue measure, both on the line (denoted by $m$ ) and in the plane (denoted by $m_{2}$ ). In order to distinguish between the two we will, when necessary, use notation like a. e. $\left[m_{2}\right]$ meaning that a property holds everywhere in the two-dimensional domain except for a set $E$ such that $m_{2}(E)=0$. Frequently, when considering a function $F(x, y)$ defined on a set $E$ in the plane, it will be useful to pass from separate measurability in each variable to measurability with respect to $m_{2}$. More precisely, with each $x$ we associate a function $F_{x}(y)$ defined by $F_{x}(y)=F(x, y)$; similarly, we define $F^{y}(x)=F(x, y)$. Sometimes, the measurability of $F_{x}$ and $F^{y}$ implies the $m_{2}$-measurability of $F$. The following elementary result (cf. [8, p. 176]) will be useful in this direction.

Lemma 2. Suppose that $F$ is a real-valued function on $\mathbb{R}^{2}$ such that each section $F_{x}$ is measurable and each section $F^{y}$ is continuous. Then $F$ is $m_{2}$-measurable.

Let $K(x, y)$ be a Lebesgue integrable function on $[0,1] \times[0,1]$. Then, it is possible to define an operator $K$ on $L^{2}(0,1)$ as

$$
K f(x)=\int_{0}^{1} K(x, y) f(y) d y .
$$


Such operators are called integral operators and we say that the function $K(x, y)$ is its kernel function, or just the kernel. We are interested in finding eigenvalues and eigenvectors for $\Phi_{V}^{K}$, the restriction of $\Phi_{V}$ to the subspace of integral operators. By Theorem 3, the set of eigenvalues of $\Phi_{V}$ is a subset of the interval $(0, \infty)$. We show that this inclusion is in fact an equality. The following result is instrumental in this direction. Namely, it gives a necessary and sufficient condition on the kernel $K(x, y)$ of an integral operator $K$ to be an eigenvector of $\Phi_{V}^{K}$. Recall that, since we assume that $\lambda$ is a positive real number, there is no loss of generality in considering the equation $V K=\beta K V$.

TheORem 6. Let $\beta$ be a positive number and let $K$ be an integral operator with kernel $K(x, y)$. Then $V K=\beta K V$ if and only if $K(x, y)=g(x-\beta y)$, for some measurable function $g$ on $\mathbb{R}$ such that $g=0$ on the interval $[-\beta, 1-\beta]$ and also $\int_{0}^{1} \int_{0}^{1}|g(x-\beta y)| d x d y<\infty$.

Proof. Let $K$ be an integral operator with kernel $K(x, y)$ and suppose that $V K f=\beta K V f$, for all $f \in L^{2}(0,1)$. Then,

$$
\int_{0}^{x} d t \int_{0}^{1} K(t, y) f(y) d y=\beta \int_{0}^{1} d t \int_{0}^{t} K(x, y) f(y) d y
$$

for all $f \in L^{2}(0,1)$ and a. e. $x$. Due to separability of $L^{2}(0,1)$ the exceptional set where (4.2) fails to hold can be chosen independent of $f$. Changing the order of integration on both sides of (4.2) yields

$$
\int_{0}^{1} f(y) d y\left[\int_{0}^{x} K(t, y) d t\right]=\beta \int_{0}^{1} f(y) d y\left[\int_{y}^{1} K(x, t) d t\right] .
$$

In view of the arbitrary choice of $f$ in $L^{2}(0,1)$ it follows that

$$
\int_{0}^{x} K(t, y) d t=\beta \int_{y}^{1} K(x, t) d t
$$

for a. e. $x$ and a. e. $y$ in $[0,1]$. Conversely, if a kernel $K(x, y)$ satisfies (4.3) for a. e. $x$ and a. e. $y$ in $[0,1]$ then it is easy to see that $V K=\beta K V$.

Suppose now that $K$ is an integral operator satisfying $V K=\beta K V$. Then its kernel $K(x, y)$ must satisfy (4.3). Notice that this kernel is indeed an equivalence class of functions that agree a. e. $\left[m_{2}\right]$. Now we pick any particular representative and denote it again by $K(x, y)$. The advantage of this strategy is that each side of (4.3) is now defined, for all $(x, y) \in[0,1] \times[0,1]$. With this understanding it is easy to see that the left hand side of (4.3), which we denote by $F_{1}(x, y)$, is a measurable function of $y$ for every $x$, and that it is a continuous function of $x$ for every $y \in[0,1]$. Hence, Lemma 2 shows that $F_{1}(x, y)$ is a measurable function with respect to the twodimensional Lebesgue measure. Since a similar argument shows that the right hand side of (4.3), which we denote by $F_{2}(x, y)$, is measurable with respect to the two- 
dimensional Lebesgue measure we conclude that $F_{1}(x, y)=F_{2}(x, y)$ a. e. $\left[m_{2}\right]$. Furthermore, $F_{1}(x, y)$ has partial derivative $\left(F_{1}\right)_{x}$ for every $(x, y) \in[0,1] \times[0,1]$ and $F_{2}(x, y)$ has partial derivative $\left(F_{2}\right)_{y}$ for every $(x, y) \in[0,1] \times[0,1]$. Hence, $F_{1}(x, y)$ has partial derivative $\left(F_{1}\right)_{y}$ for a. e. $\left[m_{2}\right]$ point $(x, y) \in[0,1] \times[0,1]$. The conclusion is that we can, and we do denote both sides of $(4.3)$ as $F(x, y)$.

A calculation shows that $F_{x}(x, y)=K(x, y)$ and $F_{y}(x, y)=-\beta K(x, y)$. Since

$$
F_{x}(x, y)=\lim _{h_{n} \rightarrow 0} \frac{F\left(x+h_{n}, y\right)-F(x, y)}{h_{n}}
$$

and each member of the sequence is a measurable function of $(x, y)$, it follows that $F_{x}$ is $m_{2}$-measurable. Similarly, $F_{y}$ is $m_{2}$-measurable. Finally, we have that

$$
F_{y}(x, y)=-\beta F_{x}(x, y), \quad \text { for a. e. }(x, y) \in[0,1] \times[0,1] .
$$

Let $(a, b)$ be any point in $[0,1] \times[0,1]$, and let $R=R_{a, b}$ denote the rectangle bounded by $x=0, x=a, y=b$, and $y=1$. Then

$$
\iint_{R} F_{x}(x, y) d x d y=\int_{b}^{1} d y \int_{0}^{a} F_{x}(x, y) d x=\int_{b}^{1} d y \int_{0}^{a} K(x, y) d x=\int_{b}^{1} F(a, y) d y
$$

while

$$
\iint_{R} F_{y}(x, y) d y d x=\int_{0}^{a} d x \int_{b}^{1} F_{y}(x, y) d y=\int_{0}^{a} d x \int_{b}^{1}-\beta K(x, y) d y=-\int_{0}^{a} F(x, b) d x .
$$

In view of (4.4), we have

$$
\iint_{R}\left[F_{y}(x, y)+\beta F_{x}(x, y)\right] d x d y=0 .
$$

Therefore,

$$
\beta \int_{b}^{1} F(a, y) d y-\int_{0}^{a} F(x, b) d x=0
$$

or, equivalently,

$$
\int_{0}^{a} F(x, b) d x=\beta \int_{b}^{1} F(a, y) d y
$$

We emphasize that (4.5) holds for every $(a, b) \in[0,1] \times[0,1]$. Denote either side of it by $G(a, b)$. Then $G$ is an $\left[m_{2}\right]$-measurable function that satisfies the equation

$$
G_{y}(x, y)=-\beta G_{x}(x, y), \quad \text { for all }(x, y) \in[0,1] \times[0,1] .
$$


Although this equation is similar to (4.4) there is an important difference. The equation (4.6) holds for every $(x, y) \in[0,1] \times[0,1]$. Now we exploit this fact by using a well known argument from classical analysis.

Let $c$ be a fixed number. The function $H(x)=G(x,(c+x) / \beta)$ is a measurable function as a composition of a measurable function $G$ and a continuous map $\rho: x \mapsto(x,(c+x) / \beta)$. Moreover, the function $H$ is a differentiable function of $x$ as a composition of two differentiable functions. Then

$$
H^{\prime}(x)=\frac{\partial}{\partial x} G(x,(c+x) / \beta)=G_{x}(x,(c+x) / \beta)+G_{y}(x,(c+x) / \beta) \frac{1}{\beta}
$$

and so, using (4.6), $H^{\prime}(x)=0$. We conclude that $H$ is a constant function of $x$. This implies that $G(x,(c+x) / \beta)$ is a constant function of $x$ so that there exists a measurable function $\tilde{g}$ such that $G(x, y)=\tilde{g}(x-\beta y)$. Since $G_{x x}(x, y)=K(x, y)$ it follows that there exists a measurable function $g$ such that $K(x, y)=g(x-\beta y)$. Moreover, the integrability condition on $K$ translates to the required integrability condition on $g$. Finally, (4.3) implies that

$$
\int_{0}^{x} g(t-\beta y) d t=\beta \int_{y}^{1} g(x-\beta t) d t
$$

for a. e. $x$ and a. e. $y$ in $[0,1]$. A change of variable leads to the equality

$$
\int_{-\beta y}^{x-\beta y} g(s) d s=\int_{x-\beta}^{x-\beta y} g(s) d s
$$

that holds for a. e. $x$ and a. e. $y$ in $[0,1]$, which in turn means that

$$
\int_{-\beta y}^{x-\beta} g(s) d s=0
$$

for a. e. $x$ and a. e. $y$ in $[0,1]$. It follows that $g=0$ on $[-\beta, 1-\beta]$.

Conversely, if $K$ is an integral operator with kernel $K(x, y)=g(x-\beta y)$, for some measurable function $g$ that vanishes on $[-\beta, 1-\beta]$, then it is easy to verify that $K$ satisfies (4.3) and the theorem is proved.

As a consequence of this result we obtain that, if $\lambda \in(0,1)$, then it is an extended eigenvalue of $V$.

Proposition 1. Let $\lambda \in(0,1)$ and let $\Delta_{\lambda}$ be the triangle bounded by $y=\lambda x$, $y=0$, and $x=1$. Also, let $K$ be the integral operator on $L^{2}(0,1)$ with kernel $K(x, y)=\chi_{\Delta_{\lambda}}(x, y)$. Then $K V=\lambda V K$.

Proof. The result follows from Theorem 6 with $g=\chi_{(0, \infty)}$ since $\chi_{\Delta}(x, y)=$ $g(x-y / \lambda)$.

When $\lambda>1$ we have to slightly change the definition of $\Delta_{\lambda}$. 
Proposition 2. Let $\lambda>1$ and let $\Delta_{\lambda}$ be the triangle bounded by $y=\lambda(x-1)+1$, $y=0$, and $x=1$. Also, let $K$ be the integral operator on $L^{2}(0,1)$ with kernel $K(x, y)=\chi_{\Delta_{\lambda}}(x, y)$. Then $K V=\lambda V K$.

Proof. Again, it is an easy application of Theorem 6 with $g=\chi_{(1-\beta, \infty)}$.

We conclude our study of integral operators satisfying the equation $K V=\lambda V K$ with the following fact that may have some independent interest.

Proposition 3. Let $\lambda$ be a positive number and let $K$ be the integral operator on $L^{2}(0,1)$ with kernel $K(x, y)$. If $K V=\lambda V K$ then there exists a nondegenerate rectangle $R=[0, a] \times[b, 1] \subsetneq[0,1] \times[0,1]$ such that $K(x, y)$ vanishes almost everywhere in $R$.

Proof. Let $\mathcal{M}$ be a nontrivial invariant subspace (n. i. s.) for $V$ and let $\mathcal{N}=(K \mathcal{M})^{-}$, where ${ }^{-}$stands for the closure in the norm topology of $L^{2}(0,1)$. It is easy to see that, in this situation, $\mathcal{N}$ is also an invariant subspace for $V$. Recall that if $\mathcal{M}$ is a n. i. s. for $V$ then there is $m \in(0,1)$ such that every function in $\mathcal{M}$ vanishes on $[0, m]$ a. e. See [9]. Similarly, since $\mathcal{N}$ is an invariant subspace (although it could be the whole $\left.L^{2}(0,1)\right)$ there is $n \in[0,1)$ such that every function in $\mathcal{N}$ vanishes on $[0, n]$ a. e.

Let $f \in \mathcal{M}$. Then

$$
K f(x)=\int_{0}^{1} K(x, y) f(y) d y=\int_{m}^{1} K(x, y) f(y) d y=\int_{0}^{1} K(x, y) \chi_{[m, 1]}(y) f(y) d y .
$$

Since $K f \in \mathcal{N}$ it follows that the last integral vanishes for a. e. $x \in[0, n]$ and every $f \in L^{2}(0,1)$. Consequently, $K(x, y) \chi_{[m, 1]}(y)=0$ for a. e. $x \in[0, n]$ and a. e. $y \in[0,1]$. Since $K(x, y)$ is $m_{2}$-measurable, it follows that $K(x, y)=0$ a. e. in $[0, n] \times[m, 1]$.

It remains to consider the case in which $n=0$. Since $\mathcal{M}$ is an arbitrary n. i. s. for $V$ we can assume that $(K \mathcal{M})^{-}=L^{2}(0,1)$, for every such $\mathcal{M}$. Let $\mathcal{M}_{1} \subsetneq \mathcal{M}_{2}$ both be n. i. s. for $V$ and let $f \in \mathcal{M}_{2} \ominus \mathcal{M}_{1}$. If $g=K f$, then there exists $h \in \mathcal{M}_{1}$ such that $K h=g=K f$. Obviously, $h-f$ is a nonzero vector in $\operatorname{Ker} K$, the nullspace of $K$. In view of $V K=\lambda K V$ we see that $\operatorname{Ker} K$ is a n. i. s. for $V$. Thus, there is $n_{0} \in(0,1)$ such that

$$
\operatorname{Ker} K=\left\{f \in L^{2}: f=0 \text { on }\left[0, n_{0}\right]\right\}
$$

Let $f \in \operatorname{Ker} K$. Then

$$
0=K f(x)=\int_{0}^{1} K(x, y) f(y) d y=\int_{n_{0}}^{1} K(x, y) f(y) d y .
$$

Since $f$ is an arbitrary function in $\operatorname{Ker} K$, the last equation shows, once again, that $K(x, y)$ vanishes a. e. in $[0,1] \times\left[n_{0}, 1\right]$. This completes the proof.

Clearly, we have just started the spectral theory for $\Phi_{V}^{K}$ and one should try to address the following problems. 
Problem 2. Describe the spectrum of $\Phi_{V}^{K}$. Describe parts of the spectrum of $\Phi_{V}^{K}$.

5. Composition operators. In this section we consider the class of composition operators on $L^{2}(0,1)$. Let $\varphi:[0,1] \rightarrow[0,1]$ be a measurable function. A composition operator $C_{\varphi}$ is defined as

$$
\left(C_{\varphi} f\right)(x)=(f \circ \varphi)(x) .
$$

We are interested in determining whether a composition operator can be an eigenvector of $\Phi_{V}$. First we notice that if the map $\varphi:[0,1] \rightarrow[0,1]$ is defined as $\varphi(x)=\lambda x$, for some $\lambda \in[0,1]$, then the composition operator $C_{\varphi}$ satisfies the equation $C_{\varphi} V=\lambda V C_{\varphi}$. Indeed,

$$
\left(C_{\varphi} V f\right)(x)=(V f)(\varphi(x))=(V f)(\lambda x)=\int_{0}^{\lambda x} f(t) d t
$$

while

$$
\left(V C_{\varphi} f\right)(x)=\int_{0}^{x}\left(C_{\varphi} f\right)(t) d t=\int_{0}^{x} f(\varphi(t)) d t=\int_{0}^{x} f(\lambda t) d t
$$

and the substitution $s=\lambda t$ yields the desired conclusion. This shows that $C_{\varphi}$ is an eigenvector for $\Phi_{V}$ corresponding to an eigenvalue $\lambda \in[0,1]$. It turns out that this example describes the only situation in which a composition operator $C_{\varphi}$ can satisfy $C_{\varphi} V=\lambda V C_{\varphi}$ or, equivalently, $V C_{\varphi}=\beta C_{\varphi} V$.

THEOREM 7. Let $C_{\varphi}$ be a composition operator on $L^{2}(0,1)$ that satisfies $V C_{\varphi}=\beta C_{\varphi} V$, for some $\beta \in(0, \infty)$. Then $\beta \geq 1$ and $\varphi(x)=x / \beta$.

Proof. Using Lemma 1 we see that $V C_{\varphi}=\beta C_{\varphi} V$ if and only if $S_{t} C_{\varphi}=C_{\varphi} S_{t / \beta}$ for all $t \geq 0$. This means that, for any $f \in L^{2}(0,1)$,

$$
\chi_{[t, \infty) \cap[0,1]}(x) f(\varphi(x-t))=\chi_{[t / \beta, \infty) \cap[0,1]}(\varphi(x)) f(\varphi(x)-t / \beta) .
$$

In particular, taking $f$ to be a constant nonzero function, we obtain

$$
\chi_{[t, \infty) \cap[0,1]}(x)=\chi_{[t / \beta, \infty) \cap[0,1]}(\varphi(x)),
$$

which shows that $x \geq t$ if and only if $\varphi(x) \geq t / \beta$. From this we conclude that $\beta \geq 1$. Indeed, if $\beta<1$, then we could take $x=t=(\beta+1) / 2$ and obtain $\varphi((\beta+1) / 2)>1$, which is a contradiction. Furthermore, it follows from (5.1) and (5.2) that, when $x \geq t, \quad \varphi(x-t)=\varphi(x)-t / \beta$. In particular, taking $x=t$, we get that $\varphi(x)=\varphi(0)+x / \beta$. However, for such a function $\varphi$,

$$
C_{\varphi} V f(x)=\int_{0}^{\varphi(0)+x / \beta} f(t) d t
$$


while

$$
V C_{\varphi} f(x)=\int_{0}^{x} f(\varphi(0)+t / \beta) d t .
$$

Using the substitution $s=\varphi(0)+t / \beta$ the last integral becomes

$$
\beta \int_{\varphi(0)}^{\varphi(0)+x / \beta} f(s) d s
$$

and it is easy to see that $\varphi(0)=0$. It follows that $\varphi(x)=x / \beta$ and the theorem is proved.

Of course, there remains the more general question that concerns the restriction $\Phi_{V}^{C}$ of $\Phi_{V}$ to the class of composition operators.

Problem 3. Describe the spectrum of $\Phi_{V}^{C}$. Describe parts of the spectrum of $\Phi_{V}^{C}$.

6. Some open problems. In Section 4 we established that the Volterra operator has a large supply of extended eigenvalues. However, this is not true for arbitrary operators with dense range. Indeed, it was shown in [2] that there are such operators for which the only extended eigenvalue is $\lambda=1$. Thus, it is of interest to characterize operators that have nontrivial (meaning different from 1) extended eigenvalues. The following questions seem natural in this context.

Problem 4. Does every compact operator have a nontrivial extended eigenvalue? Does every quasinilpotent operator have a nontrivial extended eigenvalue? What other classes have such a property?

When an operator $A$ has a nontrivial extended eigenvalue one may ask whether the extended point spectrum has any algebraic or topological structure of interest. Since in the case of the Volterra operator this set is the multiplicative group of positive real numbers one may ask whether, for example, such a set is always a semigroup. Clearly, $\Phi_{A}(I)=I$, so that 1 is an extended eigenvalue for $A$. However, when it comes to the semigroup property the situation is not clear. Indeed, let $\lambda$ and $\mu$ be two different extended eigenvalues of $A$. Then there exist nonzero operators $X$ and $Y$ such that $\Phi_{A}(X)=\lambda X$ and $\Phi_{A}(Y)=\mu Y$. Clearly, this implies that $\Phi_{A}(X Y)=$ $\lambda \mu X Y$. Unfortunately, this does not force $\lambda \mu$ to be an extended eigenvalue of $A$ since we could have that $X Y=0$. We conclude this discussion with the following question.

Problem 5. For which operators $A$ is the set of all extended eigenvalues of $A$ a unital semigroup?

AcKnowledgements. The authors would like to thank the referee for a number of important observations. In particular, his comments led to a considerable progress in Section 5. 


\section{REFERENCES}

1. H. Bercovici, Operator theory and arithmetic in $H^{\infty}$, Mathematical Surveys and Monographs No. 26 (American Mathematical Society, Providence, RI, 1988). print.

2. A. Biswas, A. Lambert and S. Petrovic, On the $\lambda$-commutativity of operators, pre-

3. S. Brown, Connections between an operator and a compact operator that yield hyperinvariant subspaces, J. Operator Theory 1 (1979), 117-121.

4. V. Lauric, Operators $\alpha$-commuting with a compact operator, Proc. Amer. Math. Soc. 125 (1997), 2379-2384.

5. V. Lomonosov, Invariant subspaces of the family of operators that commute with a completely continuous operator. (Russian) Funkcional. Anal. i Priložen. (3) 7 (1973), 55-56.

6. A. Lambert and S.Petrovic, An extension of Lomonosov's theorem, preprint.

7. C. Pearcy, Some recent developments in operator theory, Regional Conference Series in Mathematics, No. 36 (American Mathematical Society, Providence, R.I., 1978).

8. W. Rudin, Real and complex analysis, Third edition (McGraw-Hill Book Co., New York, 1987). 246.

9. D. Sarason, A remark on the Volterra operator, J. Math. Anal. Appl. 12 (1965), 244- 